and hence

$$
\alpha=D \frac{\partial \gamma}{\partial n}+\alpha \gamma
$$

This condition states that at a perfectly absorbent collector surface $(\alpha=\infty), \gamma=1$. If the surface is perfectly reflecting $\partial \gamma / \partial n=0$, which is consistent with the more natural condition $\gamma=0$.

A typical application of the formalism above would be to the diffusion of decaying particles in the presence of a radial electric field. In the earlier paper ${ }^{1}$ the formalism is applied to the very simple case of a perfectly absorbing sphere about the center of symmetry.

I would like to thank Dr. S. F. Neustadter for his interest and encouragement.

\title{
SOME PROPERTIES OF OPTIMAL LINEAR FILTERS ${ }^{1}$
}

\section{Br HERBERT A. SIMON \\ (Carnegie Institute of Technology and Cowles Commission for Research in Economics)}

The purpose of this note is to discuss two considerations that arise in designing a filter that is optimal in the sense of minimizing the integral over time of some norm, under the further condition that we do not wish to employ information about the statistical structure of the signal. This is the "classical" problem of filter design prior to Wiener's work on stationary time series, although, in general, design was and is guided by figures of merit rather than by an explicit minimization procedure. It is probable that the results set forth here are well known, at least intuitively, to designers of servomechanisms, but I have not seen them set forth.

Since the norm to be miniminized is an integral over time, the problem may be viewed as one in the calculus of variations. Suppose that the norm, $F$, is:

$$
F=\int_{t_{0}}^{t_{2}} \phi\left\{y(t), y_{(1)}(t), \cdots, y_{(n)}(t)\right\} d t,
$$

where the integrand, $\phi$, is a polynomial in some variable, $y(t)$, and its first $n$ derivatives, $y_{(k)}(t)=d^{k} / d t^{k} y(t),(k=1, \cdots, n)$; so that we may write:

$$
\phi(t)=C+\sum_{i=0}^{n} b_{i} y_{(i)}+\sum_{i=0}^{n} \sum_{j=0}^{n} a_{i j} y_{(i)} y_{(i)}+\psi(t)
$$

where $\psi(t)$ is a polynomial composed of terms of third and higher degree in $y(t)$ and its derivatives.

To obtain a necessary condition for a minimum, we form Euler's equation:

$$
\phi_{0}-\frac{d}{d t} \phi_{1}+\cdots+(-1)^{n} \frac{d^{n}}{d t^{n}} \phi_{n}=0,
$$

where $\phi_{k}=\partial \phi / \partial y_{(k)}$.

${ }^{1}$ Received April 28, 1954. The results presented in this paper were obtained in the course of research undertaken by the Cowles Commission for Research in Economics under contract Nonr-358(01), NR 047-006 with the office of Naval Research. I am indebted to my colleagues A. Charnes, C. C. Holt, and F. Modigliani with whom I have had numerous valuable discussions on the topic of this note. 
Equation (3) is a differential equation of order not greater than $2 n$ in $y(t)$. We now make the assumption:

(A). Equation (3) is a linear differential equation with constant coefficients. From (2), we obtain:

$$
\begin{gathered}
\phi_{k}=b_{k}+\sum_{i=0}^{n} a_{i k} y_{(i)}+\sum_{i=0}^{n} a_{k i} y_{(i)}+\psi_{k}(t) \quad(k=0, \cdots, n), \\
\frac{d^{k}}{d t^{k}} \phi_{k}=\sum_{i=0}^{n} a_{i k} y_{(i+k)}+\sum_{i=0}^{n} a_{k i} y_{(i+k)}+\frac{d^{k}}{d t^{k}} \psi_{k}(t) \quad(k=1, \cdots, n) .
\end{gathered}
$$

Substituting (4) and (5) in (3), we have, under assumption $(A)$ :

$$
\begin{aligned}
& b_{0}+\sum_{i=0}^{n} a_{i 0} y_{(i)}+\sum_{i=0}^{n} a_{0 i} y_{(i)}+\psi_{0}(t) \\
&+\sum_{k=1}^{n}(-1)^{n}\left\{\sum_{i=0}^{m} a_{i k} y_{(i+k)}+\sum_{i=0}^{n} a_{k j} y_{(i+k)}+\frac{d^{k}}{d t^{k}} \psi_{k}(t)\right\} \\
&=\alpha_{0} y+\alpha_{1} y_{(1)}+\cdots+\alpha_{2 n} y_{(2 n)}=0 .
\end{aligned}
$$

Rearranging the terms of (6), we obtain:

$$
\begin{aligned}
b_{0} & +\sum_{k=0}^{n}(-1)^{k}\left\{\sum_{i=0}^{n}\left(a_{i k} y_{(i+k)}+a_{k i} y_{(i+k)}\right)\right\} \\
& +\psi_{0}(t)+\sum_{k=1}^{n}(-1)^{n} \frac{d^{k}}{d t^{k}} \psi_{k}(t)-\sum_{o=0}^{2 n} \alpha_{o} y_{(o)}=0 .
\end{aligned}
$$

Now, since all the terms of $\psi$ are of third or higher degree in $y$ and its derivatives, all the non-vanishing terms of $\psi_{k}$ will be of second or higher degree, as will also all the terms of $d^{k} \psi_{k} / d t^{k}$. Hence, the linearity of the differential equation implies that:

$$
\psi_{0}(t)+\sum_{k=1}^{n}(-1)^{n} \frac{d^{k}}{d t^{k}} \psi_{k}(t)=0
$$

A necessary and sufficient condition for (8) [see Courant-Hilbert, vol. I, p. 167] is that:

$$
\int_{t_{0}}^{t} \psi\left(y, \cdots, y_{(n)}\right) d t=\xi\left(y, \cdots, y_{(n-1)}\right),
$$

i.e., that $\psi$ is independent of the path and hence does not affect the optimum.

We have proved the

Theorem I: A necessary and sufficient condition that the extremal corresponding to (2) satisfies assumption $(A)$ is that:

$$
F=\int_{t .}^{t}\left[c+\sum_{i=0}^{n} b_{i} y_{(i)}+\sum_{i=0}^{n} \sum_{i=0}^{n} a_{i i} y_{(i)} y_{(i)}\right] d t+\xi\left(y, \cdots, y_{(n-1)}\right) .
$$

From (7) we can deduce:

Theorem II: In $\sum_{g=0}^{2 n} \alpha_{s} y_{(s)}$, all $\alpha_{g}$ must vanish for odd $g$.

Proof: Since all summations in (7) run from zero to $n$, for each term $(-1)^{k}\left(a_{i k}+a_{k i}\right)$ $y_{(i+k)}$ we have a corresponding term $(-1)^{i}\left(a_{k i}+a_{i k}\right) y_{(k+i)}$. If $(i+k)$ is an odd number these terms will have opposite sign and, being otherwise identical, will vanish. 
Theorem II has an important consequence. Form the characteristic equation for ( 7$)$ :

$$
\sum_{k=0}^{n} \alpha_{2 k} p^{2 k}=0 \text {. }
$$

Because this is an even function, if $p_{1}$ is a root, $-p_{1}$ is also a root. Hence, either (1) all the roots are pure imaginaries, or (2) there is at least one root with positive real part. If we interpret the system described by the Euler equation as a linear "filter" or decision rule, the behavior produced by application of the rule will be dynamically unstable.

The two theorems we have established may be interpreted as follows:

1. From Theorem I it appears that linear filters will have desirable qualities when the norm or "error" we wish to minimize is quadratic. If the norm is decidedly nonquadratic this suggests that we can improve filter performance by introducing appropriate non-linearities.

2. From Theorem II and the consequence derived from it, it appears that straightforward application of the calculus of variations to the filter design problem leads to the prescription of an unstable filter, and hence is not practicable.

It can easily be shown that point (2) is related to the fact that the Euler equations give only a necessary and not a sufficient condition for a minimum. Hence it does not follow that a path, $y(t)$, that satisfies (3) will thereby minimize (1). The specification of appropriate initial and terminal conditions to guarantee a bona fide minimum requires information about the future of the signal, and therefore, in the face of an incompletely predictable signal the method breaks down.

The difficulty cannot be avoided by minimizing in the domain of the Laplace transform of $\phi$ instead of in the time domain. For, in general, by Parseval's theorem: ${ }^{2}$

$$
\int_{-\infty}^{\infty}[\phi(t)]^{2} d t=\int_{-\infty}^{\infty}\left[\phi^{*}(p)\right]^{2} d p
$$

where $\phi^{*}(p)$ is the transform of $\phi(t)$.

Hence, any function that is a stationary value for the right-hand side of (12) is simply the transform of the function that is a stationary value for the left-hand side.

It is beyond the scope of the present note to discuss newer methods that avoid this difficulty without reverting to cut-and-try procedures or the use of arbitrary figures of merit.

2Professor A. Charnes pointed out to me the relevance of Parseval's theorem for this problem. Minimization in the domain of the transform is essentially the method of Ritz for the "direct" solution of variational problems (Courant-Hilbert, I: p. 150).

\section{BOOK REVIEWS}

History of strength of materials with a brief account of the history of theory of elasticity and theory of structures. By S. P. Timoshenko. McGraw-Hill Book Co., Inc., New York, Toronto, London. 1953. $\mathrm{x}+452$ pp. $\$ 10.00$.

Professor Timoshenko's original contributions to the subject of Strength of Materials are outstanding in contemporary literature; the same is true of his various books on Theoretical Mechanics. It is therefore 